\title{
En torno al análisis cultural en la ciencia y la sociología política contemporáneal
}

\section{Introducción}

Tal y como el título del presente trabajo anuncia y promete, el objetivo de las siguientes reflexiones consiste en ofrecer una visión panorámica de las principales tendencias y tradiciones de pensamiento dominantes en el desarrollo de la sociología y politología contemporánea, desde una perspectiva que privilegia el análisis cultural como óptica renovadora de la producción teórica y metodológica de ambas disciplinas. La razón primordial de tal empresa radica en reconocer y revalorar, en su justa dimensión e importancia, el impresionante auge mostrado por dicha perspectiva teórica en la redefinición de los temas y problemas centrales que configuran la agenda de discusión de las ciencias sociales y políticas a lo largo de las dos últimas décadas.

En una palabra, la tarea emprendida en este espacio intenta delinear -al menos en su estructura básica - los contomos fundamentales de los problemas, intereses, enfoques y demás cuestiones de pertinencia teórico-metodológicas entre los miembros de la comunidad de investigadores, que estudian las complejas relaciones entre cultura, sociedad y política. Evidentemente, se trata de una empresa sumamente ambiciosa que, debido a la amplitud y variedad de temas, así como al hecho de la transdisciplinariedad adoptada por sus diversos enfoques de estudio, con anterioridad reconocemos y advertimos que sólo de manera parcial podrá ser alcanzada en el presente artículo.

En realidad, el asumir una perspectiva culturalista como eje del desarrollo de la sociología y la politología no representa del todo una auténtica novedad. El análisis cultural, como sabemos, constituye una perspectiva de estudio y, a la vez, un tema central para la comprensión de la naturaleza de los fundamentos de los sistemas sociopolíticos; para el estudio de sus procesos de transformación y 
cambio; para la comprensión del comportamiento de sus actores y del desempeño de sus instituciones; etcétera. Por ello, puede decirse que a pesar de los evidentes períodos de discontinuidad, abandono y marginación conceptual, la relación entre cultura y política y entre cultura y sociedad se nos ha revelado siempre como un tema viejo -o mejor dicho, clásico- de sorprendente capacidad de reactualización y rejuvenecimiento - tal y como lo puede constatar cualquier examen rápido de las respectivas etapas de las historias correspondientes de ambas disciplinas-.

De todas maneras, lo que también debe destacarse es la naturaleza paradójica y controvertida del mismo. Es decir, los estudios culturalistas - tanto en sociología como en ciencia política - ofrecen en sus resultados concretos de investigación un cierto e inequívoco carácter dual, manifestado en el hasta ahora radical contraste de, por una parte, lo rico y fructífero de algunos de sus temas y perspectivas de estudio e interpretación junto a, por otra parte, la ambigüedad y laxitud de otros.

Quizá la principal explicación de ese curioso dato provenga del carácter complejo y extenso de los fenómenos culturales, en general, y de la cultura política, en particular. Así, por ejemplo, cabe destacar que incluso hoy en día no se cuenta entre los muy diversos estudiosos de la cultura (sociólogos, antropólogos, etnólogos, politólogos) con un consenso, una definición común, compartida de cultura. Es decir, ha sido imposible, en los hechos, que los especialistas en ciencias sociales lograsen ponerse de acuerdo alrededor de un concepto fundamentado de cultura $^{2}$. En cambio, la noción de ciencia política ha corrido con mejor suerte en este sentido aunque su pecado de origen, por así decirlo, más bien ha consistido en asociarse indiscutiblemente con una particular tradición teórica (pluralista, institucionalista y de clara inspiración behaviorista) sometida en los últimos tiempos a una crítica severa - la cual tendremos ocasión de exponer en sus puntos esenciales más adelante-.

Naturalmente, ha habido muchos intentos por construir una concepción consensual, compartida de cultura. Desde la sociología existen, en este sentido, al menos cinco definiciones claras de cultura con una importante influencia en el desarrollo de la sociología contemporánea. Influencia que nos indica, por lo demás, la creciente e incuestionable legitimidad ganada por dicha perspectiva de estudio en el replanteamiento conceptual de los principales modelos explicativos predominantes en la sociología y politología de hoy. Una rápida reseña descriptiva de estas nociones nos ofrece el panorama siguiente?:

En primer lugar, la cultura entendida como una esfera especial de la realidad. Se trataría de una esfera construida a partir de la producción o creación de un conjunto definido de objetos, acciones e instituciones que abrirían a la cultura la posibilidad de un tratamiento a través de indicadores más o menos objetivo. 
En segundo lugar, tendríamos a la cultura como un contexto hermenéutico interpretativo. Según esta visión, la cultura atravesaría y marcaría con una especificidad propia a las categorías utilizadas por los sociólogos, de modo tal que la sociología más fecunda debería de adoptar la figura de una "sociología de la interpretación".

En tercer lugar, nos encontramos con la noción de cultura como variable metodológica. De acuerdo con dicha propuesta, la cultura asumiría el rol de una variable hasta ahora ausente, que obligaría revisar los principales fenómenos estudiados por la sociología. La novedad radical aquí perseguida estribaría en examinar si dichos fenómenos sufren o no transformaciones esenciales en su manifestación y/o comprensión al introducir esa nueva variable ${ }^{4}$.

En cuarto lugar, la cultura entendida como fuente de producción de la vida social. Es decir, aquí se trata de una concepción que pone su énfasis en el sentido radicalmente cultural de la vida social. Ello implica que en esta idea de cultura, a contracorriente de otras visiones predominantes, lo simbólico poseería una primacía decisiva sobre lo material.

Finalmente, tendríamos la clásica concepción marxista de cultura entendida como instrumento de dominación hegemónica. Idea que, por su familiaridad, se explicaría por sí misma.

En lo que sigue del presente artículo organizaremos nuestras reflexiones en tomo a tres apartados. Cada uno de ellos posee sobre todo un carácter analítico de corte descriptivo. El primero de ellos ofrece una suerte de mapeo general de autores y tradiciones de pensamiento en un intento omnicomprensivo (que por lo demás tampoco pretende ser exhaustivo) por indicar la amplitud y las fronteras del desarrollo del análisis cultural. En el segundo apartado, a su vez, se realiza una labor de acotación y se vuelve sobre algunos autores y escuelas que con su producción teórica han moldeado las principales problemáticas y respuestas esbozadas en dicho terreno. Finalmente, el tercer apartado quiere ofrecer un recuento de problemas, desafíos y nuevas propuestas de actualidad para intentar edificar un nuevo proyecto de cultura política.

\section{Genealogía del análisis cultural ${ }^{3}$}

El análisis cultural se encuentra ya con una presencia protagónica e instituyente desde el mismo origen del pensamiento de los padres fundadores de la sociología y la ciencia política modema. Esto es, la cultura se encuentra presente de manera inapelable en el centro del pensamiento de autores clásicos como $\mathrm{E}$. Durkheim, M. Weber y C. Marx. En el caso de Durkheim y Weber, en efecto, su herencia continúa, sin temor a exagerar, modelando, en mayor o menor medida, tanto en cuestiones metodológicas como en temas sustantivos, al conjunto del pensamiento de las ciencias sociales contemporáneas. Por ejemplo, sólo a título 
ilustrativo cabría recordar que, desde Weber, la sociología se ve atravesada por la tensión que provoca la manera de evaluar la relevancia o el rol asignado a los factores materiales e ideales en la configuración de la realidad social. En el caso de Marx, por su parte, también la influencia de su pensamiento es marcada y determinante. Aunque en este caso, sin duda por la configuración de la altura de los tiempos que vivimos, se trate más bien de una presencia orientada a reconsiderar y criticar muchas de sus tesis otrora asumidas incuestionadamente.

Si de los padres fundadores nos trasladamos hasta la primera mitad del presente siglo nos encontramos con un conocido grupo de autores que podemos denominar post-clásicos. Entre ellos cabe citar, naturalmente, a personajes de la talla de T. Parsons y A. Gramsci. Ambos, desde sus respectivas perspectivas y herencias conceptuales, formulan un claro proyecto de articulación de una "sociología de la cultura". En el caso de Parsons, poniendo énfasis en el rol protagonista de los valores a la hora de explicar la definición de la acción social. En el caso de Gramsci, en cambio, recordándonos el carácter conflictivo de las muy diversas prácticas culturales y, por ende, destacando y/o denunciando el importante papel de la cultura en los procesos societales de dominación y control.

Ahora bien, aquí merece particular mención el nacimiento, a partir de Parsons, de una corriente que fundará, propiamente hablando, el paradigma del análisis cultural desde los presupuestos de la ciencia política contemporánea. Los nombres de los principales autores post-parsonianos son ya de dominio común y dentro de ellos ocupan un puesto central G.A. Almond y S. Verba seguidos de G.B. Powell. Más adelante tendremos ocasión de volver sobre la conocida y extendida propuesta de estudio de la cultura cívica de dichos autores ${ }^{6}$. De momento, baste recordar que, a pesar de las agudas controversias y las severas críticas abiertas casi desde el inicio mismo de la formulación de dicho paradigma, el mismo continúa vigente despertando el interés y estimulando la creatividad de numerosos estudiosos a lo largo del orbe'.

Finalmente, podemos colocarnos ya en el escenario más próximo a nosotros con los autores fundamentales de las dos últimas décadas. La lista de nombres es masiva. Reiteramos que no pretendemos ser exhaustivos, sino únicamente indicativos de algunos de los más destacados autores. A todos ellos podemos englobarlos dentro de la categoría de neo-clásicos. Obviamente aquí cabe hacer unas distinciones analíticas mínimas.

Por una parte, encontramos a un grupo de pensadores que comparten su filiación neo-marxista y neo-gramsciana. Algunos nombres relevantes son los de R. Williams, R. Hoggart, E.P. Thompson y J. Habermas.

Corriendo el riesgo de caricaturizar el rico y complejo pensamiento de cada uno de esos autores podemos aceptar, no obstante, que ellos comparten algunos intereses y perspectivas básicas ${ }^{8}$. Ello les lleva, ante todo, a rechazar comprensi- 
blemente cualquier versión fuerte de determinación de la cultura concebida desde los términos de la malograda metáfora de la base y la superestructura. De igual manera, son autores que se oponen tajantemente a asignar un rol residual y reflejo a la cultura en el estudio de la dinámica societal. Desde una perspectiva más propositiva y creativa, estos autores, a su vez, reafirman su rechazo a lecturas consensuales y holistas de la cultura al propio tiempo que tratan de rescatar y resaltar, justamente, su dimensión de arena o crisol del conflicto social. De hecho, una de las más vigorosas vetas de reflexión desarrolladas desde estas posiciones es aquella que privilegia el estudio de la "cultura popular" situando su enfoque, naturalmente, en el contexto de relaciones de poder, es decir, de relaciones de dominación y resistencia.

Por otra parte, nos encontramos con un grupo de autores que, a su vez, pueden ser tipificados como neo-weberianos y neodurkheimianos. Entre los primeros destacan pensadores como C. Geertz, P. Berger, Th. Luckmann, M. Walzer y J. Gusfield. Dentro de los segundos, en cambio, encontramos figuras del tamaño de C. Lévi-Strauss, seguida de otras figuras también magistrales como $\mathbf{M}$. Douglas y $Y$. Tuimer.

Los principales rasgós compartidos por los autores neoweberianos radican en la revalorización de la dimensión intersubjetiva $y$ valorativa de la cultura. También hacen énfasis en el componente interpretativo de los símbolos $y$ su significado histórico-cultural. Ello implica que, para este tipo de pensadores, la cultura no es susceptible de ser bien entendida y manejada cabalmente de forma experimental bajo el canon y el rigor de la ciencia empírica y, por el contrario, apelan a métodos cualitativos, como el hermenéutico, para ofrecer un estudio interpretativo de fenómenos de la cultura. Por otra parte, también destacan el componente omniabarcador de la cultura y desde ahí reflexionan sobre el carácter socialmente construido del mundo social. Finalmente, los autores neoweberiamos no desatienden de sus preocupaciones teóricas - tal y como de alguna manera su tradición se los pide- el análisis de la relación entre las ideas religiosas y las prácticas políticas.

Pasando ahora a los autores neo-durkheimianos, para terminar de redondear este grueso trazado conceptual, podemos decir, a su vez, que entre otros muchos rasgos que los caracterizan y unifican podemos contar a "su atención a la naturaleza colectiva de la existencia humana, a las cuestiones del orden moral de una sociedad, a la manera en que los rituales dramatizan este orden moral y recargan a los sentimientos colectivos de una sociedad"y.

\section{Principales tendencias y desarrollos conceptuales}

Ante un panorama tan amplio de tradiciones de pensamiento, paradigmas de investigación, autores y posiciones interpretativas, resulta virtualmente imposible realizar un recuento verdaderamente exhaustivo de todas las aportaciones 
que, tanto desde la ciencia política como desde la sociología política, continúan reivindicando, hoy día, la legitimidad del uso del concepto de cultura política para alcanzar una mejor comprensión de los fundamentos de los sistemas políticos y sus procesos de transformación y cambio. Lo que, sin embargo, queda claro es la necesidad de tomar en cuenta seriamente el ininterrumpido debate abierto en tomo a la superación del paradigma clásico de cultura política. Paradigma en verdad todavía muy influyente y que, como sabernes, ostenta una clara identidad de origen de corte funcionalista $e$ institucionalista.

Este intenso debate en cuestión, por lo demás, permite distinguir al menos dos posturas en su interior. La de aquellos que sostienen críticas moderadas, permitiendo de esa manera y después de numerosas puntualizaciones y matizaciones, mantener vigente dicho paradigma, en contraposición a las posturas de los críticos radicales para quienes de lo que se trataría al respecto sería más bien de abandonar, de una buena vez, dicho enfoque y avanzar hacia una inequívoca $y$, según ellos, más fructífera "resociologización" del concepto de cultura política. En suma, si, por una parte, la noción de cultura política no ha perdido después de todo su aureola de legitimidad conceptual, desde una perspectiva más amplia persiste y se ahonda, en definitiva, el desacuerdo acerca del objeto y del alcance del análisis cultural y su exacta contribución al progreso de las ciencias sociales de hoy ${ }^{11}$.

\subsection{La tradición post-parsoniana}

Pocos conceptos han cosechado tanto éxito y despertado de manera tan incisiva el interés de los estudiosos de las ciencias sociales, como el concepto de cultura política acuñado por Gabriel Almond y Sidney Verba, a inicios de la década de los sesenta. Quizá la razón de tan marcada influencia radique en la pretensión de esa concepción por establecer una firme relación entre las actitudes y comportamientos de los integrantes de un determinado sistema político y su específico desempeño institucional. Es decir, en correspondencia con su inspiración behaviorista, la concepción de cultura política, acuñada por estos autores, adquirió identidad propia a partir de sus rasgos causalistas e instrumentales. Rasgos que permitían claramente establecer la necesidad de confianza, respeto e identificación con el sistema político por parte de sus integrantes, para garantizar la estabilidad y efectividad del mismo". No obstante, estas mismas razones que explican el éxito de tal modelo teórico justifican, de igual manera, las también numerosas reacciones críticas y de rechazo al mismo ${ }^{12}$. En realidad, el marcado y evidente sesgo ideológico inherente a dicha manera de concebir a la cultura política permitió, a diversos autores de tradiciones también diversas, enfilar hacia ella un grupo de certeras críticas, que subrayaron desde su marcado etnocentrismo hasta la denuncia del corte conservador de dicha concepción teórica.

En efecto, el componente conservador de esta concepción funcionalista de cultura política puede sostenerse a partir de varias argumentaciones, que desta- 
carían, por ejemplo, la dificultad de dicho paradigma para explicar el conflicto y el cambio social. En correspondencia con lo anterior, se puede también destacar el énfasis predominante en la creación y el sostenimiento del orden y del consenso como principales tareas asignadas a dicha noción de cultura política. Finalmente, su asociación indisoluble con las teorías de la modernización habría también contribuido a aumentar el descrédito - muy acentuado en la década de los setenta- por el paradigma en cuestión.

Otro grupo de críticas, por su parte, pusieron en cuestión el presupuesto de la existencia de una única cultura política y, por ende, el reduccionismo presente en el desconocimiento de un abanico más amplio de manifestaciones de la vida social y de estados subjetivos asociados al comportamiento político. De la misma manera, se cuestionó la pertinencia del enfoque que defiende una explicación más bien individualista de la configuración y del dinamismo manifestado por la estructura sociopolítica. En resumen, muchas otras críticas coincidieron en destacar el carácter antipolítico y las premisas antihistóricas existentes en tal concepción de cultura política.

A pesar de esta pesada batería de críticas radicales, el proyecto de Almond y Verba ha sobrevivido. Evidentemente ya no bajo el programa original estipulado en la concepción de noción de cultura cívica —dado que, inclusive, los mismos fundadores del proyecto y sus seguidores más cercanos emprendieron un serio y sincero proceso de revisión y autocrítica ${ }^{13}$ - pero sí al menos como un marco heuristico aún estimulante que sostenidamente ha impulsado el estudio de la cultura politica. En concreto, puede decirse que los puntos fuertes, que en cierta manera sobreviven a la crítica radical, serían, en el plano normativo, la idea de que la cultura puede transformarse en normas sociales con todo lo que de explicación del comportamiento político ello implica y, en el plano metodológico, la afirmación del valor positivo del uso de una metodología empírica para su estudio.

En definitiva, parece quedar claro que "no existen culturas que por definición nomativa sean más favorables para la estabilidad de la democracia, (Y) tampoco existe un conjunto de actitudes que puedan constituir una precondición para la existencia de la democracia"14. Por supuesto, las premisas anteriores de ninguna manera invalidan el claro hecho de que el estudio del compartimento político posee, en sí mismo, un lugar de primera importancia en la politología contemporánea, por cuanto ésta se preocupa por medir e interpretar el peso y significado de las actitudes individuales y de grupo, así como la naturaleza de su incidencia en el funcionamiento y calidad de los sistemas políticos.

\subsection{La tradición neo-marxista}

La otra tradición conceptual que ha realizado una lectura marcadamente política de la influencia de los fenómenos cultural en el interior de la sociedad y, en concreto, de su impacto en la definición y el dinamismo del comportamiento 
socio-político es, naturalmente la tradición neo-marxista. Dentro de ella encontramos, como ya advertíamos líneas arriba, importantes diferencias de énfasis que determinan, por así decirlo, su sello característico en el entendimiento de la mediación entre cultura y acción social.

En concreto, en el caso de la tradición neo-gramsciana, todo su análisis sobre el fenómeno cultural parte del presupuesto que concibe al todo social como una arena de conflicto. Ello implica que la manifestación de cualquier proceso cultural tienen lugar en el marco de sociedades profundamente divididas. Por ello, la cultura debe concebirse como parte de un proceso de dominación.

Bajo tales presupuestos se rompe tajantemente con cualquier lectura convencional del papel asignado a la cultura política. Así, a diferencia de los enfoques tradicionales (como el de los post-parsonianos), que asignan un papel de integración y estabilidad social a la cultura, aquí se afirma la tesis totalmente opuesta. Es decir, la tesis de que la cultura es socialmente desintegradora e inevitablemente fuente permanente de conflictos sociales.

En realidad, a los seguidores de este planteamiento no se les escapa el paradójico hecho de que la cultura es, al mismo tiempo, fuente de integración y de desintegración; de asociación y de disociación. De esta suerte, si ellos se preocupan por destacar el componente conflictivo de la cultura se debe a que consideran que en la mayoría de los casos, cuando la cultura es utilizada con fines de integración a lo que se llega es a formas patógenas y artificiales de convivencia social ${ }^{15}$.

Además, la principal ventaja de hacer visibles las diferencias interculturales (y su evidente componente de incommensurabilidad) ${ }^{16}$ consiste en colocar a la producción y reproducción del poder en el centro del análisis cultural. Ello implica ver en las manifestaciones culturales un medio que contribuye significativamente a la organización de la sociedad, desde la afirmación de intereses, capacidades, orientaciones, valoraciones marcados por la diferencia y el conflicto. Se trata, por tanto, de una lectura más apropiada y realista del dinamismo sociocultural y desde donde se abre el desafío de explicar la estabilidad y el cambio de manera más congruente con dicho principio ${ }^{17}$.

Desde la variante neo-marxista, el planteamiento más interesante (y también en cierto sentido controvertido) sobre la naturaleza, los alcances y el rol de la cultura política es el desarrollado por J. Habermas a lo largo de su extensa obra ${ }^{18}$. En su pensamiento sobre el tema, sobre todo en una etapa temprana del mismo, Habermas concibe a la cultura política como expresión y manifestación de la racionalidad burguesa. En este sentido, Habermas imprime una interpretación de corte estructural e histórico a su concepción de cultura política, la cual objetiviza por medio del estudio de su manifestación en la así denominada esfera pública.

En efecto, la noción de esfera pública hace referencia a la existencia de un espacio social en donde se producen y contraponen los discursos de los indivi- 
duos y grupos que componen a la sociedad. Por ello le compete, por su propia definición, el convertirse en un espacio de manifestación de la vida cívica de cada sociedad. Es decir, los principales problemas políticos de la sociedad y, en suma, la manifestación espontánea de su propia vida comunitaria autogestiva definen, en consecuencia, los contenidos y los dinamismos de la esfera pública. De hecho, la palabra esfera es una suerte de metáfora para describir y ubicar un escenario estructural (locus) integrado por instituciones y redes discursivas en donde se objetiviza la cultura política de las sociedades industriales avanzadas. En este sentido, forma parte de su naturaleza más íntima la ausencia del control directo del aparato estatal y de sus mecanismos y procedimientos coercitivos ${ }^{14}$. Queda claro, por tanto, que la sustancia de la esfera pública se deriva y está orientada hacia la manifestación de la sociedad civil, de modo que puede sostenerse que de la existencia de una vigorosa sociedad civil -participativa, deliberativa - es válido deducir la existencia de un ordenamiento institucional realmente democrático.

De manera demasiado resumida, sin duda, hemos presentado la noción de esfera pública en el pensamiento habermasiano. Una concepción que comparte con la previa postura neomarxista presentada acá la afirmación del componente comunicativo -y, por ende, de poder- presente en los símbolos culturales, así como de la necesidad de su adecuado enraizamiento y/o contextualización histórica para captarlos en su pleno significado.

\section{Hacia un nuevo proyecto de cultura política}

Después de nuestro muy sintético y, además, pretendido omniabarcador recorido doctrinario, salta a primera vista que en el campo del análisis cultural existen una cantidad impresionante de problemas teórico-conceptuales y prácticos de gran envergadura. Se trata de problemáticas inherentemente complejas para las que, con franqueza, no existen respuestas sencillas ni mucho menos ampliamente admitidas.

En este sentido, queda claro, por ejemplo, que en el caso del influyente paradigma de la ciencia política empírica, uno de los principales retos ha sido el de superar sus planteamientos de inmutabilidad y de determinismo de los valores y de la cultura. Mientras que, también a título ilustrativo, en el caso de las visiones ortodoxas de la teoría de la elección racional, ha sido igualmente constante el desaflo de superar los postulados, economicistas e individualistas de los intereses.

Lo cierto es que más allá de esas intrínsecas dificultades y los desafiantes retos presentes en este terreno, las diversas escuelas y tradiciones de pensamiento continúan asumiendo la tarea de perfeccionar sus propuestas de análisis de la cultura, en general, y de la cultura política, en particular, en cuanto variable central en la interpretación del dinamismo de la acción social. No existe, en efecto, un proyecto altemativo y novedoso para el estudio de la cultura completamente consolidado y coherente. Existen, eso sí, distintas propuestas —algunas complementa- 
rias, otras más bien confrontadas entre sí- con distintos niveles de progreso, de originalidad y de pertinencia, pero todas ellas están orientadas a alcanzar tal objetivo renovador. En semejante empresa colectiva algunas de esas posturas, que destacan por el interés que despiertan y el potencial que prometen, son:

(1) El ponerse como objetivo fundamental la tarea de hallar una instancia teórica que sea capaz de vincular la estructura y el actor o la cultura y el actor manteniendo su autonomía, pero también su interdependencia. Únicamente dentro de dicho marco es concebible una adecuada explicación de las relaciones establecidas entre las ideas y sus respectivas influencias en los individuos.

(2) Comprender la cultura como un conjunto de herramientas (integrada por símbolos, tradiciones y concepciones de mundo) que proporcionan a los individuos y grupos determinadas "estrategias de acción". En tal perspectiva, el papel asignado a la cultura justamente consiste en proporcionar los recursos que necesitan los actores para definir su acción social. La ventaja de comprender la cultura bajo estos términos estriba en superar el determinismo causalista, que plantea que los valores moldean a la acción social por una visión flexible según la cual la cultura proporciona los insumos (entendidos como instrumentos y no como contenidos), pero no determina mecánicamente el comportamiento societal ${ }^{211}$.

(3) Colocar al estudio de las preferencias en el centro del análisis cultural, en tanto que la expresión de las mismas constituye el fundamento de la acción política. Es decir, asumir como presupuesto que el origen de nuestras preferencias reside en el contexto de las relaciones sociales que nos envuelve y prefigura. En este sentido, la manifestación de preferencias contienen un componente endógeno, por cuanto toda elección está determinada por contextos culturales - no estructurales - entendidos como modos de vida de los actores sociales ${ }^{21}$.

(4) Encontrar o construir un retomo a la historia. Es decir, el estudio de la interacción entre cultura y estructura social debe ser planteado en términos de análisis histórico. Ello con el propósito de encontrar un método explicativo englobante y común a la determinación que las circunstancias estructurales ejercen sobre los sistemas culturales y -viceversa - entender cómo los sistemas culturales son capaces, a su vez, de organizar determinados tipos de acción social que modifican los dinamismos estructurales de la sociedad.

(5) Finalmente, en el plano metodológico la postura, quizá ya no tan novedosa pero todavía muy controvertida, radica en sostener la necesidad de admitir una transformación y relativización del uso de encuestas de opinión como la mejor manera de estudiar la cultura política. El presupuesto teórico que sostiene tal postura considera que la cultura no es un conjunto homogéneo y bien trabado de actitudes valores y creencias acerca de la realidad política, que es compartido por el conjunto de una población nacional dada. Al contrario, dicho presupuesto sostiene que en la manifestación de la vida sociopolítica coexisten diferentes culturas, las cuales no necesariamente tienen que poseer un indicador empírico signi- 
ficativo común. De ahí que se juzgue como más apropiado para el análisis cultural la incorporación o introducción de técnicas sociológicas de carácter cualitativo.

\section{A manera de conclusión}

Cerramos esta visión panorámica del estado actual del análisis cultural en la sociología y la ciencia política contemporánea con una cita de María Luz Morán quién considera que dicho análisis "se enfrenta a la tarea de ir recogiendo aportaciones de una amplísima literatura y aplicar diversas propuestas a uno de los campos que desde el origen de la disciplina suscitó un interés cognoscitivo fundamental: determinar la influencia de los universos políticos de los individuos en la naturaleza funcionamiento y cambio de los sistemas políticos. Los interrogantes y las áreas de sombra siguen siendo, sin duda, muy numerosos. Pero las perspectivas abiertas son apasionantes ${ }^{22}$ ". Conclusión general que nosotros también plenamente compartimos.

\section{Notas}

1. Ponencia presentada en el Segundo Congreso Nacional de Ciencia Política, realizado en la ciudad de México del 7 al 9 de octubre de 1998. El autor desea agradecer a la Secretaría de Relaciones Exteriores por el apoyo institucional brindado, sin el cual no habría sido posible la participación en el presente evento.

2. A1 respecto, Nikdas Luhmann dice: "el espectro del concepto (de cultura) se ha extendido tanto que bien puede considerarse ya demasiado amplio. Abarca desde los fundamentos simbólicos de la acción (Parsons), hasta la totalidad de los artefactos humanos. Incluye las máquinas electrónicas hasta los tatuajes en el cuerpo humano; altas culturas y culturas cotidianas; cultura de las tribus arcaicas y cultura de las sociedades modernas ... entonces ya casi no se pueden establecer límites en lo social. C fr. N. Luhman, "La cultura como concepto histórico", Mimeo.

3. A1 respecto, véase C. Calhoun, Critical Social Theory, Cambridge, Blackell, 1995, citado por J. Auyero en "La cultura que vuelve. Una revisión de las perspectivas de análisis cultural en la sociología norteamericana”, en Debates en Sociologia (1996), 20-21, pp. 73-75.

4. Un muy buen ejemplo de esta manera de entender el desafío teórico que implica incorpora a la cultura como "variable" ausente en muchos y muy influyentes análisis de las ciencias sociales hoy día lo encontramos en el caso de la teoría de la elección racional. Sus nociones centrales -interés, calculo, individuo, etcétera-, ciertamente, son profundamente alteradas al tomar en serio la dimensión cultural. En este sentido, cabe destacar la contribución de Jon Síster por formular una teoría de la elección racional con apego a valores. Se trata de una contribución que, por lo demás, no escapa a la controversia. Algunas de sus principales obras en esta línea de reflexión son: J. Síster, Juicios Salomónicos: las limitaciones de la racionalidad como principio de decisión, Barcelona, Gedisa, 1991. Y, también, El cemento de la sociedad. Las paradojas del orden social, Barcelona, Gedisa, 1992.

5. La presente propuesta genealógica de autores y escuelas reproduce con ligeras variaciones la desarrollada por J. Auyero, en la obra arriba citada. Otras propuestas taxonómicas del desarrollo del análisis cultural, aunque desde el acotado punto de vista de la 
ciencia política y que puede guardar algún interés para el lector, pueden encontrarse en Almond G., A Discipline divided. Schools and secta en political sciencie, Newbury Park, Sage, 1990, especialmente el Cap. 5, "The study of political culture", pp.139156. Véase también A. Sermeño, "Sobre cultura y participación política", en Realidad. Revista de Ciencias Sociales y Humanidades, 1996, 49, pp. 81-103.

6. Las obras clásicas de dichos autores, en donde abordan directamente el desarrollo del tema de la cultura política, son: G.A. Almond y S. Verba, The Civic Culture. Political Attitudes and Democracy in Five Nation, Princeton, Princeton University Press, 1963; The Civic Culture Revisited, Sage, Newbury Park, 1989.

7. Para obtener una visión de síntesis de los debates y las controversias desarrolladas alrededor de la propuesta de la cultura cívica, puede consultarse $\mathrm{M}$. Torcal, "Cultura política", en R. del Águila (ed.), Manual de Ciencia Politica, Madrid, Trotta, 1997, pp. 231-250.

8. Cabe destacar que para todo el contenido de la presente sección se han simplificado, por comodidad expositiva, los rasgos conceptuales compartidos por los autores pertenecientes a cada escuela o tradición mencionada. Un análisis más detallado de cada uno de ellos, incluso en el interior de una misma tradición, nos revelará, sin duda, que a pesar de compartir una misma influencia conceptual, la mayoría de los autores también revelan significativas dif erencias entre sí.

9. J. Auyero, "la cultura que vuelve"... op. cit., p. 89.

10. M.L. Morán, "Sociedad, cultura y política: continuidad y novedad en el análisis cultural", en M.L. Morán (comp.), Zona abierta (número monográfico dedicado al tema Cultura y Política), 77-78, 1996/97, pp. 1-30.

11. En términos generales, desde la tradición pluralista-institucional, se acepta por cultura política al conjunto de "crecncias, valores y capacidades que son comunes al total de la población así como también a las tendencias especiales, modelos y patrones que sólo pueden encontrarse en sectores particulares de esta última". Además, aparejados al concepto de cultura política se encuentran también los conceptos de socialización y secularización política. El primero dado que por su propia naturaleza engloba al proceso de introyección de determinadas actitudes políticas y valores. El segundo, por su parte, debido a que se refiere a la modernización del sistema político, mediante "el proceso por el cual los hombres acrecientan la capacidad racional, analítica y empírica de su acción política. Cabe destacar que desde dicha perspectiva de estudio, la comprensión del fenómeno cultural se encuentra sesgada por el persistente empeño por establecer el tipo de relación existente cntre, por una parte, las orientaciones subjetivas de una determinada población nacional y, por otra, el desempeflo gubernamental, social y económico manifestado por la misma. Al respecto puede consultarse O.A. Almond y G.B. Powell, Política comparada: una concepción evolutiva, Buenos Aires, Paidós, 1972. Asimismo G.A. Almond y S. Verba, The Civic Culture, op. cit.

12. M. Torcal, op. cit., pp. 235-241.

13. Una primera reacción correctiva del proyecto original de la cultura política muy anticipado al de Civic Culture Revisited se encuentra en L.W.

14. M. morcal, op. cit., p. 245.

15. La brutal explosión de rabiosos nacionalismos, las crecientes prácticas xenofóbicas o la extendida ola de intolerancias que recorre al mundo en la recta final del siglo XX constituyen, en esta perspectiva, la mejor ilustración del carácter patógeno con que operan los mecanismos artificiales de integración cultural. 
16. La inconmensurabilidad de la cultura manifiesta el irreductible hecho según el cual, más allá de cierto espacio de encuentro entre las culturas, se llega a un límite en donde las diferencias entre ellas se vuelven insuperables. De ahí que el respeto a las diferencias culturales suponga un gran desafío teórico y conceptual al pensamiento democrático liberal contemporáneo. Justificar y generar esquemas institucionales de convivencia intercultural, sin romper con el universalismo del discurso democrático, es el reto asumido por el ala más progresista del pensamiento liberal hoy en día. Al respecto, véase por ejemplo J. Rawls, Liberalismo político, México, FCE, 1995; Will Kymlicka, Ciudadonía multicultural, Barcelona, Paidós, 1996; Ch. Taylor, El multiculturalismo y la Política del reconocimiento, México, FCE, 1993.

17. Como nos dice K. Eder: "el problema general que se esconde tras esta propuesta es si una sociedad ha de ser pensada como un orden social o como un contexto interactivo dinámico; o mejor aún, si el diseño y el contraste puede convertirse en principio organizativo de la producción y reproducción social". K. Eder, "La paradoja de la "cultura". Más allá de una teoría de la cultura como factor consensual, en M.L. Morán (comp.), Zona Abierta, op. cit., p. 122.

18. A pesar de la amplia bibliografía habermasiana, los textos que contienen su concepción seminal de cultura política como esfera pública son: Transformación estructural de la esfera pública, Barcelona, 1981, y su Teoría de la acción comunicativa, Madrid, Taurus, 1987 (dos volúmenes). Asimismo cabe destacar que el tema es retomado y replanteado en numerosas y sucesivas obras de la "industria" de producción teórica, en que se transmuta el mismo Habermas.

19. Dice Habermas: "la esfera o espacio de la opinión pública es, ciertamente, un fenómeno social tan elemental como la acción, el actor, el grupo o el colectivo; pero escapa a los conceptos tradicionales de orden social. La esfera o espacio de la opinión pública no puede entenderse como institución y, ciertamente, tampoco como organización; no es un entramado de normas con diferenciación de competencias y de roles, con regulación de las condiciones de pertenencia, etc.; tampoco representa un sistema; permite, ciertamente, trazados internos de límites, pero sc caracteriza por horizontes abiertos, porosos y desplazables hacia el exterior. El espacio de la opinión pública, como mejor puede describirse es como una red para la comunicación de contenidos y tomas de posturas, es decir, de opiniones, y en él los flujos de comunicación quedan filtrados y sintetizados de tal suerte que se condensan en opiniones públicas agavilladas en tomo a temas específicos", J. Habermas, Facticidad y validez, Madrid, Trotta, 1998, p. 440.

20. La autora que defiende esta propuesta es Ann Swidler. Véase, al respecto, A. Swidler, "La cultura en acción: símbolos y estrategias", en M.A. Morán (comp.), Zona Abierta, op. cit., pp. 127-162.

21. Véase, al respecto, A. Wildavsky, "La elección de preferencias a través de la construcción de instituciones", en M.A. Morán, Zona Abierta, op. cit., pp. 163-197.

22. M.L. Morán, "Sociedad, cultura y política...." op. cit., p. 26. 